\title{
BIFURCATING ATTRACTORS AND GALERKIN APPROXIMATES
}

\author{
R. WELLS AND J.A. DUTTON
}

Let $u=A_{0} u+\mu A_{1} u+J(u)$ be a Navier-Stokes parameterized evolution equation in a Hilbert space $H$ and let $F_{1} \subset F_{2} \subset F_{3} \subset \ldots$ be an increasing sequence of finite dimensional spaces such that every $F_{n} \oplus \mathbb{R}$ contains the center-unstable linear subspace $H^{u} \oplus \mathbb{R} \subset H \oplus \mathbb{R}$ of the system $\dot{u}=A_{0} u+\mu_{1} u+J(u), \dot{\mu}=0$. Then each $F_{n} \oplus \mathbb{R}$ determines a Galerkin approximant of the original system, with the same center-unstable linear subspace $H^{\mathcal{H}} \oplus \mathbb{R}$. The flow on the center-unstable manifold of the original system may be identified with a parameterized flow on $H^{\mathcal{U}}$ given by $x=f_{\infty}(x, \mu)$. The flow on the center-unstable manifold of the Galerkin approximant determined by $F_{n} \oplus \mathbb{R}$ may be identified with a parameterized flow on $H^{\mu}$ given by $\dot{x}=f_{n}(x, \mu)$. It is proved that Theorem 1 holds: $\lim _{n \rightarrow \infty} f_{n}=f_{\infty}$ in the $c^{k}$ topology on a compact neighborhood of the origin in $H^{\mathcal{H}} \oplus I R$. From this theorem it is concluded that Theorem 2 holds: If a certain priori bound

Received 23 April 1986. We would like to thank Professor Michael Shub, CUNY, for drawing our attention to the results of $F$. Wesley Wilson III on Lyapunoy functions, Professor Peter Kloeden, Murdoch University, for keeping us informed of his own work on this subject (Kloeden [8]), and Professor Rouben Rostamian, Penn State University, for a very helpful conversation. We would also like to thank the National Science Foundation for support through Grant ATM 8307213.

$\$$ A2.00 +0.00 .

Copyright Clearance Centre, Inc. Serial-fee code: 0004-9727/87 
holds relating $f_{\infty}$ and $f_{n}$ and an asymptotically stable set $A$ of $\dot{x}=f_{n}(x, \mu)$ near the origin, then $\dot{x}=f_{\infty}(x, \mu)$ has an asymptotically stable set near the origin with the same Borsuk shape as $A$. Conversely, for each asymptotically stable set near the origin of $\dot{x}=f_{\infty}(x, \mu)$, there is one of the same Borsuk shape for $\dot{x}=f_{n}(x, \mu)$ provided $n$ is large enough. Informally, these results amount to the statement that asymptotically stable sets of the Navier-stokes equation, bifurcating from a steady solution, are recovered up to Borsuk shape by those of large enough Galerkin approximants.

\section{Introduction}

The method of truncated models, or Galerkin approximants, is a much used practical technique for investigating the evolution of hydrodynamic and thermo-hydrodynamic systems near steady solutions. (Dutton [4], Dutton and Wells [5].)

A typical example of such a system is given by the Boussinesq equations for an incompressible fluid box heated from below.

$$
\begin{aligned}
& \partial u / \partial t=\partial P / \partial x+\sigma \Delta u-\nu \cdot \operatorname{grad}(u) \\
& \partial v / \partial t=\partial P / \partial y+\sigma \Delta v-v \cdot \operatorname{grad}(v) \\
& \partial w / \partial t=\partial P / \partial z+\sigma \Delta w+\theta-\nu_{\nu} \cdot \operatorname{grad}(w) \\
& \partial \theta / \partial t=\Delta \theta+\sigma \lambda w=v_{\nu} \cdot \operatorname{grad}(\theta) \\
& 0=\partial u / \partial x+\partial v / \partial y+\partial w / \partial z
\end{aligned}=N\left[\begin{array}{l}
u \\
v \\
w \\
\theta \\
P
\end{array}\right]
$$

Here $v=(u, v, w)$ describes the velocity of the fluid at a given point, $\theta$ the temperature and $P$ the pressure, $\sigma$ the Prandtl number and $\lambda$ the rate of heating. We impose free, conducting boundary conditions by requiring that

$$
0=w-\partial u / \partial z=\partial v / \partial z \text { at } z=0 \text { and } z=1
$$

and

$$
\theta=T_{0} \text { at } z=0 \text { and } \theta=T_{1} \text { at } z=1
$$

so that $\lambda$ is proportional to $T_{1}-T_{0}$. If the box is described by 
$0 \leq z \leq 1,0 \leq x \leq 2 d_{1}$ and $0 \leq y \leq 2 d_{2}$, and we impose periodic boundary conditions on the vertical boundaries, then the 5-tuples of the form

$$
\left[\begin{array}{c}
u \\
v \\
w \\
\theta \\
P
\end{array}\right]=\left[\begin{array}{l}
\operatorname{trig}_{1}(x, y, z) \\
\operatorname{trig}_{2}(x, y, z) \\
\operatorname{trig}_{3}(x, y, z) \\
\operatorname{trig}_{4}(x, y, z) \\
\operatorname{trig}_{5}(x, y, z)
\end{array}\right]
$$

form a basis $B$ for a Hilbert space in which the dynamical system (1) may be presumed to evolve, where

$$
\text { (1.5) } \begin{array}{r}
\operatorname{trig}(x, y, z)=[\sin \text { or } \cos ]\left(\pi a_{1} x / d_{1}\right) \cdot[\sin \text { or } \cos ]\left(\pi a_{2} y / d_{2}\right) \cdot \\
{[\sin \text { or } \cos ](\pi n z)}
\end{array}
$$

with $a_{1}, a_{2}$, and $n$ integral. A steady solution of (1) is given by the zero 5-tuple; a member of the Hilbert space near that solution is given by

$$
\xi=\Sigma\{c(\varepsilon) \varepsilon \mid \varepsilon \text { in } B, \quad c(\varepsilon) \in \mathbb{R}\}
$$

with

$$
|\xi|^{2}=\Sigma c(\xi)^{2} a(\xi)
$$

small, where the numbers $a(\varepsilon)$ are suitable positive weights. A finite truncated model attempting to describe the evolution of small perturbations of the zero solution is constructed as follows. First one selects a finite vector subspace $F$ of the Hilbert space. Then any member $\xi$ may be written uniquely

$$
\xi=T_{F} \xi+C_{F} \xi
$$

with $T_{F} \xi$ in $F$ and $C_{F} \xi$ orthogonal to $F$. Now, the system determined by 
(1.9)

$$
\left[\begin{array}{l}
\partial \xi_{1} / \partial t \\
\partial \xi_{2} / \partial t \\
\partial \xi / \partial t \\
\partial \xi_{4} / \partial t \\
0
\end{array}\right]=T_{F} N(\xi) \text { for } \xi \text { in } F
$$

evolves in a way that one hopes will reflect in some way the evolution of the system (1). We say that (1.9) is the Galerkin or finite truncated model determined by the subspace $F$. One choice for the subspace $F$ is the subspace spanned by the 5-tupules.

(1.10) $\left[\begin{array}{l}\sin \left(\pi x / d_{1}\right) \cos (z) \\ 0 \\ -\left(\pi / d_{1}\right) \cos \left(\pi x / d_{1}\right) \sin (z) \\ 0 \\ 0\end{array}\right]\left[\begin{array}{l}0 \\ 0 \\ 0 \\ \cos \left(\pi x / d_{1}\right) \sin (z) \\ 0\end{array}\right]\left[\begin{array}{l}0 \\ 0 \\ 0 \\ \sin (2 z) \\ 0\end{array}\right]$

The resulting truncated model is the celebrated Lorenz model.

The Lorenz model [10] appears to possess a strange attractor; in fact, Williams' geometric model of the Lorenz model of the Lorenz system does contain a strange attractor (Williams, [18]). Furthermore, higher order truncated models of various thermo-hydrodynamic systems generate highly suggestive but non-conclusive numerical evidence pointing to the presence of strange attractors (Nese [12]). Thus, one may expect that the presence of strange attractors in these finite truncated models eventually may be rigorously established.

The central problem in this area is whether the original hydrodynamic and thermo-hydrodynanic system itself possesses strange attractors. And, unfortunately, even if the presence of strange attractors is established in an associated finite model, one cannot conclude a priori that the original system contains these attractors because the truncation obscures the relations between the two systems. Thus we arrive at the problem of deciding when the presence of a strange attractor in a suitable finite truncated model implies the presence of a similar attractor in the original 
system.

Having isolated the latter problem, we must bring it into a more specific and manageable form. To do so, we examine two issues: the definition of the notion of a strange attractor and the relevance of a nearby steady solution. To deal with the first issue, we restrict ourselves for the moment to finite dimensional flows and note that even for these the appropriate definition of an attractor is still not clear. The broadest definition is a partly measure theoretic one and is given by Guckenheimer and Holmes [6]. This definition requires an attractor to be an invariant set posessing a dense orbit and a basin of positive measure. What is intended is that an attractor be an irreducible invariant set detectable by an iterative numerical approximation of the flow. Since our methods are not measure theoretic, we turn to an alternate detection procedure, which is still computable in principle. This procedure is to build polyhedra $P$ with the property that the flow at any point of the frontier $F P$ of $P$ is directed into the interior of $P$. Then the object of interest is the $\omega$-limit set contained in $P$. Of course, such an $\omega$-limit set is a compact asymptotically stable set; conversely, a theorem of Wilson [19] implies that a compact asymptotically stable set is contained in a trapping manifold: A trapping manifold is a smooth compact submanifold $M$ of the phase space, having the same dimension as the phase space and having the property that at any point of the boundary $\partial M$ of $M$ the flow is directed into the interior of $M$. From such a trapping manifold, traingulation theorems (Hirsch and Mazur [7]) may be used to construct the polyhedron $P$. Thus, compact asymptotically stable sets are exactly the sets detected by such polyhedra.

What is the relationship between attractors in the sense of Guckenheimer-Holmes and asymptotically stable sets? Clearly any one of the latter is one of the former, but Guckenheimer-Holmes attractors may possess infinitely generated cohomology while asymptotically stable sets always have the Borsuk shape (Borsuk [2]) of polyhedrons, and thus, cohomology of finite type. In particular a set which is minimal in the class of asymptotically stable sets need not be minimal in the class of Guckenheimer-Holmes attractors. An example of an attractor with infinitely generated cohomology is the solenoidal flow described by Poston and stewart 
[14] while in an example of an asymptotically stable set is Williams' geometric Lorenz attractor (Williams [18]). In fact, this Lorenz attractor has the shape of a figure eight, and williams describes it as the intersection of the forward iterates of a polyhedral basin. In general an asymptotically stable set containing a dense orbit contains no proper asymptotically stable subsets; Williams' geometric Lorenz attractor is such a set. Now, a reasonable conjecture is that a Guckenheimer-Holmes attractor is a countable nested intersection of a asymptotically stable sets containing it. The solenoidal flow of Poston and stewart is such an attractor and the remarkable curve of Birkhoff [1] appears to be such an attractor. If this conjecture is valid, then an asymptotically stable set which contains a dense orbit is minimal also in the class of Guckenheimer-Holmes attractors. In any case, we will regard asymptotically stable sets, their shapes and cohomology groups as justifiable objects of interest in the study of dynamical systems, and it is the appearance of these in the infinite dimensional dynamical system that we will seek to infer from their appearance in suitable finite truncated models of that system.

The second issue that we encounter is the need for a nearby steady solution. This need is dictated by our technique, which is to apply the Center Manifold Theorem. Rather than to attempt to define a reasonable concept of nearness, we include the requirement of nearness by focusing our attention on bifurcation of asymptotically stable sets from steady solutions. In this case we obtain our result, spelled out in greater detail in the next two sections.

THEOREM. In a Navier Stokes dynomical system, if an asymptotically stable set bifurcates from a steady solution, then an asymptotically stable set of the same Borsuk shape bifurcates from the corresponding steady solution in any sufficiently large finite trumcated model.

This theorem is the principal consequence of the more technical Theorem 1 in section 2. A partial converse to the theorem above also appears in the section as Theorem 2. Here our intention is only to clarify, with minimum technicality, the claim being made in the theorem above, and thus in the theorems of Section 2 . 
There are two issues involved in the clarification of the theorem above, the meaning of Borsuk shape and the role played by the possible breakdown of strong solutions of the Navier Stokes equations.

The technical definition of Borsuk shape is to be found in [2]. It is not necessary for the purpose of this paper to repeat that complicated definition here, but only to characterize the notion of Borsuk shape for asymptotically stable sets. A characterization is the following:

Borsuk Shape Two asymptotically stable sets, possibly in distinct dynamical systems, have the same Borsuk shape if and only if their basins have the same homotopy type.

This characterization is all we will use to prove the theorem above. To illustrate the notion of shape for the convenience of the reader, we point out that a single point, a circle and a figure eight all have distinct shapes; the Lorenz attractor has the shape of a figure eight; an Ostlund-Sethna-Siggia-Rand [13] wrinkled torus has the shape of an ordinary 2-torus; and the solenoidal attractor described by poston and stewart [2] has a shape distinct from all the foregoing. Finally, any contractible compact space has the shape of a point.

As suggested above, Cech cohomology groups are natural invariants of shape. A simpler invariant is the Euler Number $E(A)$ of a compact space $A$ - characterized as follows:

1. If $A$ and $B$ are compact spaces of the same shape, then $E(A)=E(B)$.

2. If $A$ is a contractible compact space, then $E(A)=1$.

3. If $A$ and $B$ are compact subspaces of the same space, then $E(A \cup B)=E(A)+E(B)-E(A \cap B)$.

With this well-known characterization of the Eulex Number, it is easy to distinguish the shapes of many simple spaces such as spheres and polyhedrons. For each example, the reader may calculate in a few steps that the Euler Number of a point is 1 , that the Euler Number of a circle is 0 , and that the Euler Number of a figure eight is -1 . 
The value of the notion of shape arises from the fact that it affords a means for a coarse classification of attractors. Of course, the Euler Number affords the means for an even coarser classification of attractors. For example, if the Euler Number of an attractor is known to be -1 , then it cannot possibly be a closed orbit.

Besides that of the meaning of Borsuk shape, the second issue that the theorem above raises is the problem of the possible breakdown of strong solutions. Here we remark that for the hypotheses of the Weissler Theorems [17] (which we describe in Section 3 and thereafter) to be satisfied, our Hilbert space $H=H_{4}$ in Section 4 must be at least the classical space $V$ in which strong solutions reside, or a subspace of a higher Sobolev space. Thus, strong solutions may well break down after a finite time depending on the $V$-norm of the initial condition. However, our asymptotically stable sets are compact, as guaranteed for example by Constantin, Foias and Temam [3]. Sufficiently small invariant neighborhoods are then bounded in the $V$-norm so that the $V$-norm of a solution in such a neighborhood cannot become infinite. Then a result of Temam [16] implies that the solution is defined for all positive times. Thus it is legitimate to speak of asymptotically stable sets in $V$ an higher (divergence free) sobolev spaces.

Finally, we remark that the approach of this paper was inspired by the presentation of Lanford's proof of the Center Manifold Theorem in Marsden-McCracken [11]. Since that book appeals to the Weissler Theorems rather than the more classical Foias-Temam development, so do we. Consequently, sections 3 to 5 contain recollections of this known material and the necessary adaptation of that material to our purpose. The new material - the proof of our theorems - begins in Section 6, but we doubt that it can be read without Sections 3 to 5 . Unfortunately, this approach results in the theorems not being expressed in terms of the standard space $V$, but the translation is easily made.

\section{The Theorems}

If we eliminate the pressure term from a Navier-Stokes thermohydrodynamic system in the usual way, we arrive at an evolution equation of the form 


$$
\begin{aligned}
& \dot{u}=A_{0} u+\mu A_{1} u+J(u) \\
& \dot{u}=0
\end{aligned}
$$

with $A_{0} u, A_{1} u$ and $J(u)$ defined for all $u$ in a pre-Hilbert space $H_{0}$ (which is a subspace of the $C^{\infty} 4$-vector fields) with norm $\mid$. The term $J(u)$ is contributed by the quadratic non-linearities in the NavierStokes equations, so we may assume that $0=\left.\frac{d}{d s}\right|_{0} J(s u)$. We have chosen a smooth steady solution as the origin of coordinates so that there is no constant term. Finally, $\mu$ is a system parameter such as the Rayleigh number.

Still preserving a fair degree of generality, we may assume that $H_{0}$ is the algebraic direct sum of the eigenspaces of $A_{0}$, all finite dimensional and all but finitely many corresponding to eigenvalues with negative real parts. Let $H^{c}$ be the direct sum of the eigenspaces corresponding to eigenvalues of $A_{0}$ with non-negative real parts. In the next section we will impose further conditions sufficient to ensure that (2.1) has a solution local semigroup $\Phi_{t}(u, \mu)=\left(\phi_{t}(u, \mu), \mu\right)$ in $H \oplus \mathbb{R}$, and that this in turn has a center-unstable manifold $M$ at the origin: $M$ is a $c^{k}$ submanifold (for some $k$ ) of $H \oplus \mathbb{R}$, invariant under the local semigroup, whose tangent space at the origin is $H^{c} \oplus \mathbb{R}$; it is not necessarily unique. It is known that $M$ is locally attracting near the origin with respect to the local semigroup $\Phi$; that is there exists $\alpha>0$ and for each $T$ there exists $\varepsilon>0$ such that ||$x||+|\mu|<\varepsilon$ implies that $\Phi_{t}(x, \mu)$ is defined for $0 \leq t \leq T$ with

$$
\text { distance }\left(\Phi_{t}(x, \mu), M\right) \leq \varepsilon a^{-t a}
$$

Thus it makes sense to identify the "local asymptotic behavior" of $\Phi$ near the origin with that of $\Phi$ restricted to $M$. Without defining explicitly what this behaviors may be (different centre unstable manifolds would seem to lead to different such behaviors, unless we make the definition carefully), we see that what we are really looking for is the restriction of $\Phi$ to one center-unstable manifold; such a restriction suffices to 
determine the local asymptotic behavior. Since the germ at the origin of an invariant set is common to all centre-unstable manifolds at the origin, any one of these suffices to determine it. Any bifurcation that takes place at $(u, \mu)=(0,0)$ is determined by such a germ; thus we may regard in particular any bifurcation at $(u, \mu)=(0,0)$ as part of the local asymptotic behavior of $\Phi$ near the origin, which is completely determined by any single center-unstable manifold.

Since $\Phi$ restricted to $M$ is a $c^{k}$ local semi-flow on a finite dimensional $c^{k}$ manifold $M$, it is completely determined by its $\left(c^{k-1}\right)$ vector field $\tilde{L}(u, \mu)$ for $(u, \mu) \in M$. Let $\pi: H \rightarrow H^{c}$ be orthogonal projection. Then there is a neighborhood $U$ of the origin in $H^{C} \oplus \mathbb{R}$ on which a $C^{k-1}$ vector field $L$ is well-defined by the characterization

$$
(\pi \oplus i d) \tilde{L}(u, \mu)=L(\pi(u), \mu)
$$

The local semiflow $\Psi$ of $L$ is equivalent to that $\tilde{L}$ that is $\Phi \mid M$, via $\pi \oplus$ id, so the local asymptotic behavior of $\Psi$ is equivalent to that of $\Phi \mid M$ and so, in turn to that of $\Phi$. Finally then, it is the $c^{k-1}$ vector field $L$ on the open subset $U$ of $H^{C} \oplus \mathbb{R}$ that we seek.

We wish to determine $L$ as the limit in the $c^{k-1}$ topology of $c^{k-1}$ vector fields $L^{n}$ on $U$ arising from finite dimensional systems. These systems will be certain Galerkin approximants of $(2,1)$ in $H$. We recall how to construct a Galerkin approximant of a system such as (2.1): we let $F$ be a finite dimensional subspace of $H_{O} ;$ we let $\pi_{F}: H \rightarrow F$ be the orthogonal projection and we consider the system on $F \oplus \mathbb{R}$ given by

$$
\begin{aligned}
& \dot{v}=\pi_{F}\left(A_{0} v+\mu A_{1} v+J(v)\right) \\
& \dot{\mu}=0
\end{aligned}
$$

This system is called the Galerkin approximant of (2.1) determined by the sub-space $F$. Clearly, if $F \supset H^{C}$, then the tangent space to the centreunstable manifold of (2.4) at the origin will be precisely the same as that of $\Phi$, namely $B^{C} \oplus I R$. Then the solution local semi-flow of (2.4) will 
determine a $c^{k-1}$ vector field $L_{F}$ on a neighborhood of the origin in $H^{c} \oplus I R$ in exactly the same way that $\Phi$ leads to $L$. To determine our vector fields $C^{n}$ with $C^{k-1}$ limit $L$, we let $F^{0} \subset F^{1} \subset F^{2} \subset \ldots$ be a sequence of finite sums of eigenspaces of $H_{O}$, with

$$
H^{c}=F^{0}
$$

and

$$
\stackrel{\infty}{n}=0^{n} F^{n}=H_{0}
$$

We set $L^{n}=L_{F^{n}}$. Then our main result is given by the following theorem.

THEOREM 1. If the system (2.1) satisfies in addition conditions i), ii), iii), and $i v$ ) of Section 4, then for any $k<\infty$ there exists an open neighborhood $U$ of the origin in $H^{C} \oplus \operatorname{IR}$ such that:

a) The fields $L, L^{1}, L^{2}, L^{3}, \ldots$ are all defined in $U$.

b) $L=\lim _{n \rightarrow \infty} L^{n}$, where the limit is taken in the $c^{k-1}$ topology.

We may assume that $U$ is a compact rather than open neighborhood.

To examine the content of this Theorem, consider an asymptotically stable invariant set $A$ for the first line of (2.1) and $\mu=\mu_{0}$.

Suppose that $A$ and $\mu_{0}$ are sufficiently close to 0 so that $A \times \mu_{0} \subset \operatorname{int}\left(\pi^{-1}(U)\right)$. Since then $A \times \mu_{0} \subset M \cap \pi^{-1}(U)$, we may identify $A \times \mu_{0}$ with corresponding invariant set in $U_{\mu_{0}}=\left\{x \mid\left(x, \mu_{0}\right) \in U\right\}$. Let fields $L_{\mu_{0}}$ and $L_{\mu_{0}}^{n}$ on $H^{c}$ be defined by $L\left(x, \mu_{0}\right)=\left(L_{\mu_{0}}(x), 0\right)$ and $L^{n}(x)=\left(L_{\mu_{0}}^{n}(x), 0\right)$. Since $A \times \mu_{0}$ is asymptotically stable, it follows from wilson [19] that there exists a trapping manifold $N$ for $A$ in $U_{\mu_{0}}$ for the flow $L_{\mu_{0}}$. Recall that $N$ is a smooth compact manifold with bounday, of dimension equal to that of $H^{c}$, with $L_{\mu_{0}}$ directed into $N$ 
at every point of $\partial N$. Since both $A$ and $N$ are compact, asymptotically stable sets of the flow of $L_{\mu_{0}}$, both with the same basin, it follows that the Borsuk shape of $A$ is that of $N$.

On the other hand, since $L_{\mu_{0}}^{n}$ approximates $L_{\mu_{0}}$ in the $c^{k-1}$ topology, $N$ will be a trapping manifold for the flow of $L_{\mu_{0}}^{n}$ once $n$ is large enough. We conclude that for large $n$ the Galerkin approximant determined by $F^{n}$ contains an asymptotically stable set of the same shape as $A$. In particular, for example, both will have the same Cech cohomology groups.

More generally, if an attractor $A$ is the intersection of a countable nest of asymptotically stable sets, any initial segment of the nest may be recovered up to shape as above in sufficiently large Galerkin approximants, so that the shape of $A$ (and thus its cohomology) is approximated as well as one pleases by that of asymptotically stable sets in Galerkin approximants.

The problem remains of deciding which trapping manifolds of the flow of $L_{n}$ will not contribute to the cohomology of asymptotically stable invariant sets of the flow of $L$ (and thus, of the flow of the first part of (2.1) with $\mu$ fixed). We evade this problem by reducing it to knowledge of an a-priori bound on $\left|L-L_{n}\right|_{U}$, where we use the version of Theorem 1 with $U$ compact and ||$_{U}$ denotes the $C^{0}$ norm of vector fields on $U$. Then the following theorem holds.

THEOREM 2. Let $N$ be a trapping manifold in the interior of $U_{\mu_{0}}$ for the flow defined on $H^{c}$ by $L_{\mu_{0}}^{n}$. Suppose that for every $x \in \partial N$ the inequality

$$
\left|L_{\mu_{0}}^{n}(x) \cdot u(x)\right|>\left|L-L^{n}\right|_{U}
$$

where the dot indicates inner product and $u$ is the inward vonit normal 
field along $2 N$. Then the flow of equation (2.1) contains an asymptotically stable set with the shape of $N$.

\section{Weissler's Theorem}

We will make use of the Existence and Uniqueness of F. Weissler [17] and an immediate corollary. In this section we state that theorem and draw the corollary.

The theorem deals with an initial value problem in Hilbert space

$$
\dot{u}(t)=A u(t)+J u(t) ; u(0)=x \text {. }
$$

The operator $A$ is the infinitesimal generator of a $C_{0}$ semigroup $e^{t A}$ on $E$, and $J$ is a non-linear function on a subset of $E$ with $J(0)=0$. In addition the following hypotheses are made:

\section{Hypotheses}

For every $t>0$, the map $e^{t A} J$ extends to a $C^{\infty}$ map $E \rightarrow E$.

With $B(\rho)$ the closed ball of radius $\rho$ in $E$ and $c_{\rho}(t)$ equal to the Lipschitz constant of $e^{t A} J$ on $B(\rho)$, for every $\rho>0$ there is some $\varepsilon>0$ such that the function $c_{\rho}(\cdot)$ is in $L^{1}(0, \varepsilon)$.

Now we may state the theorem.

WEISSLER'S THEOREM. If the hypotheses above hold, then there exists a vonique local semiflow $\phi_{t}$ on $E$ such that

a) For $u(t)=\phi_{t^{\alpha}}$ and $0 \leq t \leq T_{\alpha}=$ existence time of $\phi_{t^{\alpha}}$,

$$
u(t)=e^{t A} \alpha+\int_{0}^{t} e^{(t-s) A} J(u(s)) d s .
$$

b) If $v:[0, T] \rightarrow E$ is strongly measurable, essentially bounded, and satisfies (3.2), then $v(t)=u(t)$.

c) If the existence time $T_{\alpha}$ is finite, then $|u(t)| \rightarrow \infty$ as $t \rightarrow T_{\alpha}$.

d) If $e^{t A} J$ extends to a globally Lipschitz map, then $T_{\alpha}=\infty$ for all $\alpha$ and $\phi_{t}$ is a global semigroup. 
We will regard (3.2) as the statement that $u(t)$ satisfies (1.1). In fact (1.1) implies (3.2); Weissler proves the converse in a strong sense in the case that $e^{t A} J: E \rightarrow E$ is continuously Frechet differentiable; this condition we subsume here into the hypothesis that $e^{t A} J: E \rightarrow E$ be $C^{\infty}$.

We will need a corollary of Weissler's Theorem in the following environment:

i) $\quad F$ is another Hilbert space and $1: F \rightarrow E$ is a compact embedding.

ii). An operator $A_{F}$ on $F$ is the infinitesimal generator of a $C_{O}$ semigroup $e^{t A} F$ on $F$, such that

$$
\imath \circ e^{t A} F=e^{t A} \circ \imath
$$

iii) $e^{t A} F$ 。 extends to a $C^{\infty}$ map, also denoted by

$$
e^{t A} F_{J}: E \rightarrow E
$$

iv) The latter map $e^{t A_{F}}$ is globally Lipschitz for all $t$ with $c(\cdot)$ in $L^{1}(0, \varepsilon)$ as above.

It follows from an application of Weissler's Theorem that we have two local semigroups $\phi_{t}^{E}$ and $\phi_{t}$, in $E$ and $F$ respectively, such that

$$
\phi_{t}^{E} \circ \imath=\imath \circ \phi_{t}^{F}
$$

For $u^{E}(t)=\phi_{t}^{E}(r(\alpha))$ and $u^{F}(t)=\phi_{t}^{E}(\alpha)$ we have

$$
u^{E}(t)=e^{t A}(u(\alpha))+s_{0}^{t} e^{(t-s) A} J\left(u^{E}(s)\right) d s
$$

and

(3.7)

$$
\left.u^{F}(t)=e^{t A}(\alpha)+s_{0}^{t} e^{(t-s) A} F_{J(l}\left(u^{E}(s)\right)\right) d s
$$


In addition, the existence time $T_{B}^{E}$ in $E$ is infintie for all $B$. Now we may state the corollary we will need.

COROLLARY TO WEISSLER'S THEOREM. The existence time $T_{\alpha}^{F}$ in $F$ is also infinite for all $\alpha$.

Thus both $\phi_{t}^{E}$ and $\phi_{t}^{E}$ are global semigroups.

Proof of Corollary. Conclusion b) of Weissler's Theorem implies that i० $u_{F}(t)=u_{E}(t)$ for $0 \leq t \leq T_{\alpha}^{F}$. Conclusion c) implies that $\left|u^{F}(t)\right|_{F} \rightarrow \infty$ as $t \rightarrow T^{F}$. Then (3.7) implies that

$$
\left|s_{0}^{t} e^{(t-s) A_{F}} J\left(u_{E}(s)\right) d s\right|_{F} \rightarrow \infty
$$

at $t \rightarrow \frac{F}{T_{\infty}}$. However, our hypothesis iii), together with the continuity of $u(t)$ implies that

$$
t \rightarrow s_{0}^{t} e^{(t-s) A_{F}} J\left(u_{E}(s)\right) d s \text { in } F
$$

is a continuous function of $t$ for all $0 \leq t<T_{\alpha}^{E}=\infty$, which cannot approach $\infty$ as $t \rightarrow T_{\alpha}^{F}<\infty$.

\section{Existence and uniqueness by Weissler's Theorem}

To prove Theorem 1 , we shall need fairly versatile guarantees of existence and uniqueness of solution local semi-groups for systems like (2.1). These are given by Weissler's Theorem [17]. With these in hand, we may adapt Lanford's proof of the Center-Unstable Manifold Theorem as presented in Marsden-McCracken [11] to the case of a flow such as that of (2.1). The linearization at $\infty$ in that proof works because of Weissler's Theorem, and the contraction mapping in that proof appears here as a contraction semi-group. The center-unstable manifold in Lanford's proof corresponds to a fixed point of the contraction mapping. Since here we have a contraction semi-flow, the centre-unstable manifold corresponds here to a solution of a partial differential equation with singular 
boundary conditions; the solution is unique (in the right non-linear function space) since the fixed point of a contraction is unique. The same procedure may be carried out for the Galerkin approximants of Section 2 to produce solutions for their corresponding partial differential equations. Now we apply the classic device for existence theorems in fluid dynamics (Ladyzhenskaya [9]): The function space that contains all the solutions of the partial differential equations is a bounded subset of a Hilbert space; the common domain of these functions is an open subset of the finite dimensional $H^{c} \oplus \mathbb{R}$, and the functions are at least differentiable on that domain. The boundedness and the cantor process enable us to select a sequence of solutions weakly convergent on a countable dense subset of the domain; a compact map makes the convergence strong; the local equicontinuity resulting from the differentiability enables us to apply a suitable variant of the Arzela-Ascoli Theorem to obtain strong convergence. A few more compact maps enable us to improve the convergence further, so that it is compatible with the partial differential equations, and the limit of solutions is a solution. Finally, it is this last fact, suitably interpreted, that implies immediately the convergence in Theorem 1 .

To carry out the program above in some what greater detail, we begin by arranging our hypotheses so that Weissler's Theorem applies. At this point we may as well arrange for the occurrence of the compact maps and smoothness that we need later in the argument. Our hypotheses then are the following:

i) We are given four Hilbert completions $H_{1}, H_{2}, H_{3}, H_{4}=B$ of $H_{0}$, with the last being the completion of the original metric, together with continuous linear maps

$$
H_{1} \stackrel{\mathfrak{l}_{1}}{\rightarrow} H_{2} \stackrel{\mathrm{l}_{2}}{\rightarrow} H_{3} \stackrel{\mathrm{l}_{3}}{\rightarrow} H_{4}=H
$$

induced by the identity on $H_{0}$ and which preserve orthogonality; moreover, ${ }^{2} 1$ is to be compact. Let their norms be denoted by ||$_{i}$ for $i=1,2,3,4$. Normally, the $H_{i}$ are Sobolev spaces. 
ii) On each of these spaces $A_{0}$ extends to an operator with compact resolvent and $A_{1}$ to a bounded operator. Further, we assume that $A_{0}$ and $J$ extend to $C^{\infty}$ maps $H_{i} \rightarrow H_{i+1}$ for $i=2,3$. We do not introduce notation to distinguish the different extensions of the same operator, but allow the context to determine them.

iii) $A_{0}$ is the generator of a $C^{0}$ semigroup on each of the spaces $H_{i}$ for $i=1,2,3,4$,

$$
e^{t A_{0}}: H_{1}+H_{i}
$$

iv) for $t>0$ and $g: H_{4} \rightarrow I R$ a $C^{\infty}$ function, the map $u \rightarrow e^{t A} 0\left(\mu A_{1} u+g(u) J(u)\right)$ extends to a $C^{\infty}$ map $H_{4} \rightarrow H_{1}$. Let $c_{i}(\alpha, \mu, g, t)$ for $i=1,2,3,4$ be the corresponding Lipschitz constant for the map on the $\alpha$-balls of $H_{i}$. We assume that for some $\varepsilon>0$ and all $\alpha>0, c_{i}(\alpha, \mu, g, \cdot) \in L^{1}(0, \varepsilon)$.

In the case of fluid mechanics, $A_{0}$ is essentially the Laplacian ${ }^{t A} O$ is the solution to the heat equation. Since the latter is and $e=$ is the solution to the heat equation. Since the latte
infinitely smoothing for $t>0$ and since $J(u)$ is the standard advection term, a quadratic form that drops differentiability by one, this hypothesis is easily verified, though it appears complicated.

An immediate consequence of these hypotheses, given by Weissler's Theorem, is that the system (2.1) has unique solution local semigroups in $H_{i} \oplus I R$ for $i=1,2,3,4$. Since these are unique, it follows that they are compatible with the maps $L_{i}$ for $i=1,2,3$.

Finally, we will need to replace these local semiflows with global ones. We choose $0<\varepsilon_{1}<\varepsilon_{2}$ and we let $\gamma: \mathbb{R} \rightarrow \mathbb{R}$ be a non-increasing $C^{\infty}$ function such that

$$
\gamma(r)=1 \text { for } r \leq \varepsilon_{1}
$$


and

(4.4)

$$
\gamma(r)=0 \text { for } \varepsilon_{2} \leq r \text {. }
$$

Then we observe that the non-linear operator given by

$$
u \rightarrow e^{t A}\left(\mu A_{1} u+\gamma\left(|u|_{4}^{2}\right) J(u)\right)
$$

is globally Lipschitz on $H_{4}$. We may conclude from Weissler's Theorem that there exists a unique global semiflow for $i=4$

$$
\Phi_{i}^{t}: H_{i} \oplus I R \rightarrow H_{i} \oplus I R
$$

where

$$
\Phi_{i}^{t}(x, \mu)=\left(\phi_{i}^{t}(x, \mu), \mu\right)
$$

solving the evolution equation

$$
\dot{u}=A_{\mu} u+\gamma\left(|u|_{4}^{2}\right) J(u)
$$

$$
\dot{\mu}=0
$$

in the Hilbert space $H_{4}$. The corollary to Weissler's Theorem then implies the same for $i=1,2,3$ as well.

Since (2.1) and (4.8) are identical near the oxigin of $H=H_{4}$, they will have the same invariant subspaces near the origin, and the same center-unstable manifolds. Thus, we may as well assume from now on that we are dealing with (4.8) rather than (2.1). Notice that outside a bounded set in $H_{4},(4.8)$ agrees with the linear part of (2.1); this is the generator of the "linearization at $\infty$ " of the solution local semigroup of (2.1) corresponding to Lanford's linearization.

\section{Lanford's Argument}

Lanford's proof of the Center-Unstable Manifold Theorem applies to a diffeormorphism rather than to a flow. The first step is to linearize the diffeomorphism outside a ball; we have taken the corresponding step by 
replacing (2.1) with (4.8).

We now translate his argument to our setting. Let $H_{i}^{c}$ be the closed subspace of $H_{i}$ spanned by the generalized eigenspaces of $A_{0}$ corresponding to eigenvalues with real part non-negative, and let $H_{i}^{S}$ be the closed space spanned by those corresponding to eigenvalues with real part negative. Then we may write for $u \in H_{i}$,

$$
u=v \oplus w
$$

with $v \in H_{i}^{c}$ and $w \in H_{i}^{\mathbf{s}}$, and

$$
\phi_{i}^{t}(v \oplus w, \mu)=\alpha_{i}^{t}(v \oplus w, \mu) \oplus B_{i}^{t}(\nu \oplus w, \mu)
$$

or

$$
\Phi_{i}^{t}(v \oplus w, \mu)=\left(\alpha_{i}^{t}(v \oplus w, \mu) \oplus B_{i}^{t}(v \oplus w, \mu), \mu\right)
$$

with $\alpha_{i}^{t}(\nu \oplus w, \mu) \in H_{i}^{c}$ and $\beta_{i}^{t}(v \oplus w, \mu) \in H_{i}^{s}$

Define for some $r \geq 2$

(5.4) $V_{i}^{r}=\left\{f: H_{i}^{c} \circ \mathbb{R} \rightarrow H_{i}^{s} \mid f\right.$ is $c^{r},\left|D^{j} f(y)\right| \leq 1$ for all $0 \leq j \leq r$ and $\left.y \in H_{i}^{c} \circ \mathbb{R} ; \quad f(0)=0, \quad D f(0)=0\right\}$

where $D$ is the Jacobian differential operator.

We wish to define for each $t \geq 0$ a mapping $p_{i}^{t}: v_{i}^{r} \rightarrow v_{i}^{r}$ by requiring that

$$
\phi_{i}^{t}(v \oplus f(v, \mu), \mu)=\alpha_{i}^{t}(v \oplus f(v, \mu), \mu) \oplus\left(p_{i}^{t} f\right)\left(\alpha_{i}^{t}(v \oplus f(v, \mu), \mu), \mu\right),
$$
or, equivalently, that

$$
\left(p_{i}^{t} f\right)\left(\alpha_{i}^{t}(v \oplus f(v, \mu), \mu), \mu\right)=\beta_{i}^{t}(v \oplus f(v, \mu), \mu) .
$$

As in Marsden-McCracken [11], we may check that for a fixed $k \geq 3$, if $\varepsilon_{3}$ and $t$ are small enough, then $p_{i}^{t}: V_{i}^{r} \rightarrow v_{i}^{r}$ is defined and contracting 
for all $r \leq k$.

So far, we have merely replaced the diffeomorphism in Lanford's proof with the local semigroup solving (2.1), and the linearization at $\infty$ of that diffeomorphism with the global semigroup $\Phi_{i}^{t}$, which is sometheing like the linearization at $\infty$ of the solution local semiflow of (2.1). Then the contraction map $p_{i}^{t}: v_{i}^{r} \rightarrow V_{i}^{r}$ of Lanford's proof appears here with a parameter $t$, apparently constrained to small values of $t$. However, the global semigroup property of $\Phi_{i}^{t}$, which follows from Weissler's Theorem, implies that

$$
p_{i}^{t} \circ p_{i}^{s}=p_{i}^{t+s}
$$

whenever both sides make sense. From (5.7) and the fact that $p_{i}^{t}$ is globally defined, we may define $p_{i}^{t}$ for all $t$, so that $p_{i}^{t}$ is a global contraction semigroup on $v_{i}^{r}$ for $r \leq k$.

Since $p_{i}^{t}$ is a global contraction semigroup, it will have a unique fixed point $f_{i}^{\infty}$ in $v_{i}^{k}$, and the graph

$$
\Gamma\left\{\left(f_{i}^{\infty}\right)=\left(v \oplus f_{i}^{\infty}(v, \mu), \mu\right) \mid v \in H_{i}^{c}, \mu \in \mathbb{R}\right\}
$$

is the $c^{k}$ center-unstable manifold we seek. Furthermore, since $v_{i}^{k}$ is an invariant subspace of $v_{i}^{r}$ for $r=1, \ldots, k$ under the semigroup $p_{i}^{t}$, we see that the fixed point of $p_{i}^{t}: v_{i}^{r} \rightarrow v_{i}^{r}$ is $f_{i}^{\infty}$, independent of $r=1, \ldots, k$.

\section{An infinite dimensional PDE}

The advantage we gain by our alteration of Lanford's argument is that $p_{i}^{t}$ is a global semigroup. From this fact and equation (5.6) we see that $f=f_{i}^{\infty}$ in $V_{i}^{p}$ if and only if $f$ satisfies 
(6.1)

$$
\left.\frac{d}{d t}\right|_{0} f\left(\alpha_{i}^{t}(v \oplus f(v, \mu)), \mu\right)=\left.\frac{d}{d t}\right|_{0^{\beta}} ^{t}(v \oplus f(v, \mu), \mu) .
$$

We re-write (4.8) in terms of $H_{i}^{c}$ and $H_{i}^{S}$ components,

$$
\begin{aligned}
& \dot{v}=B_{\mu} v+M(v, w) \\
& \dot{w}=c_{\mu} w+N(v, w)
\end{aligned}
$$

with $M$ and $N$ the non-linear parts, satisfying

$$
\begin{aligned}
M(0) & =0 \\
N(0) & =0 \\
D M(0) & =0 \\
D N(0) & =0
\end{aligned}
$$

Then, as in Guckenheimer and Holmes [6, p.131] (6.1) becomes the partial differential equation

$$
D_{v} f(v, \mu) \cdot\left[B_{\mu} v+M(v, f(v, \mu)]=C_{\mu} f(v, \mu)+N(v, f(v, \mu))\right.
$$

with singular boundary conditions

$$
f(0)=0, \quad D_{v} f(0)=0
$$

Here $D_{v}$ denotes the Jacobian differential operator with respect to $v$. Thus we conclude that $(6.4-6.5)$ has a unique solution in $v_{i}^{r}$, and that this solution is $f_{i}^{\infty}$.

Notice that the domain of $f$ in (6.4-6.5) is finite dimensional, and that it is only the range which is infinite dimensional.

In view of the identity

$$
H_{1}^{c} \oplus \mathbb{I R}=H_{i}^{c} \oplus \mathbb{I R} \text { for } i=1,2,3,4
$$

we may regard (6.4-6.5) as a partial differential equation on $H_{1}^{C} \oplus \mathbb{R}$ with values in $H_{i}^{s}$ for $i=1,2,3,4$. The uniqueness of the solutions $\Phi_{i}^{t}$ implies that they are compatible: it follows then that the semigroups $p_{i}^{t}$ 
are compatible. Thus

$$
\text { (6.7) } \quad 1_{i} \circ f_{i}^{\infty}=f_{i+1}^{\infty} \text { for } i=1,2,3 \text {. }
$$

Consequently we may abuse notation slightly and write $f^{\infty}=f_{i}^{\infty}$ for $i=1,2,3,4$ from now on. Also, we write $H^{c}$ for $H_{1}^{c}=H_{2}^{c}=H_{3}^{c}=H_{4}^{c}$ from now on.

7. The Galerkin approximants and their center-unstable manifolds

By taking sums of generalized eigenspaces of $A_{0}$ in $H_{0}$, we may construct a sequence of finite dimensional vector subspaces of $H_{0}$.

$$
\text { (7.1) } \quad F^{1} \subset F^{2} \subset F^{3} \subset \ldots
$$

invariant under $A_{0}$ and having dense union in $H_{i}^{s}$. Let

$$
q_{i}^{n}: H_{i}^{s} \rightarrow F^{n}
$$

be the orthogonal projection. Of course

$$
q_{i}^{n}=q_{i+1}^{n} \circ 1_{i}
$$

The finite dimensional vector space $H^{\mathcal{H}} \oplus F^{n} \oplus \mathbb{R}$ determines a Galerkin approximant of (4.8) near the origin, which in the form of (6.4) is given by

(7.4)

$$
\begin{aligned}
& \dot{v}=B_{\mu} v+M(v, w) \\
& \dot{\omega}=C_{\mu}^{n} w+N^{n}(v, w) \\
& \dot{\mu}=0
\end{aligned}
$$

where

$$
c_{\mu}^{n}=q_{i}^{n} \circ c_{\mu}
$$

and

$$
N^{n}(v, w)=q_{i}^{n} N(v, w)
$$

for $i=1,2,3,4$. Because of (7.3), the Galerkin approximants obtained from any $B_{i}$ are the same and we may omit the subscript. 
By repeating Lanford's argument and introducing a partial differential equation as in Sections 5 and 6 for the system (7.4), we conclude that a center-unstable manifold of (7.4) is given by the graph

$$
\Gamma\left(f^{n}\right)=\left\{\left(v \oplus f^{n}(v, \mu), \mu\right) \mid v \in H_{i}^{c}, \mu \in \mathbb{R}\right\},
$$

where $f^{n}$ is the unique $c^{k}$ solution with derivatives bounded by 1 of the singular first order problem

$$
\begin{aligned}
D_{v} f^{n}(v, \mu) \cdot\left[B_{\mu} v\right. & +M\left(v, f^{n}(v, \mu)\right]=c_{\mu}^{n} \cdot f^{n}(v, \mu) \\
& +N^{n}\left(v, f^{n}(v, \mu)\right) f^{n}(0)=0, D_{v} f^{n}(0)=0 .
\end{aligned}
$$

Now, the functions $f^{n}: H^{c} \oplus I R \rightarrow F^{n}$ may be regarded as functions $f^{n}: H^{c} \oplus \mathbb{R} \rightarrow H_{i}^{\mathcal{S}}$, and so, as members of $V_{i}^{k}$. But then for $j=0,1, \ldots, k-1$ and $i=1$, the sequence of $j$ th derivatives

$$
D^{j} f^{1}, D^{j} f^{2}, D^{j} f^{3}, \ldots
$$

is strongly equicontinuous. In order to make use of this fact, we make two simple observations for a finite dimensional space $F$, Hilbert spaces $H$ and $H^{\prime}$, and $L(F, H)$ the Banach space of linear maps $F \rightarrow H$ :

I) Since $L(F, H)$ is isomorphic as a Banach space to $H \oplus \ldots \oplus H$ with $\operatorname{dim} F$ terms, the unit ball in $L(F, H)$ is weakly compact.

II) Suppose that $H^{\prime} \rightarrow B$ is compact; since the induced map $L\left(F, H^{\prime}\right) \rightarrow L(F, H)$ is essentially the map $H^{\prime} \oplus \ldots \oplus H^{\prime} \rightarrow H \oplus \ldots \oplus H$, it too is compact.

Now, given any subsequence of (7.9), using (I), we may select a subsequence which converges weakly on the rational points of $H^{c} \oplus \mathbb{R}$. Regarding the maps $f^{h}$ now as maps into ${B_{2}^{c}}_{2}$, using (II) and the compactness of $H_{1} \rightarrow H_{2}$, we see that our second subsequence now converges strongly on the rational points of $H^{C} \oplus \mathbb{I R}$. Moreover, regarded as arising from maps into $H_{2}^{c}$, our original sequence (7.9) is even more strongly equicontinuous than it is when regarded as arising from maps into 
$H_{1}$. Thus, we are in a position to apply the Arzela-Ascoli Theorem to conclude that our second subsequence converges strongly on all of ${ }_{H}^{f} \oplus I R$ when it is regarded as arising from maps into $H_{2}^{c}$. Thus, any subsequence of the sequence

$$
f^{1}, f^{2}, f^{3}, f^{4}, \ldots: B^{U} \oplus \mathbb{R} \rightarrow H_{2}^{c}
$$

contains a subsequence $h^{1}, h^{2}, h^{3}, \ldots$ converging strongly to a $c^{k-1}$ function $h \in V_{2}^{k-1}$.

Consider such a sequence,

$$
f^{n \ell}=h^{\ell}: H^{c} \oplus \mathbb{R}+F^{n} \ell \text {, }
$$

with $n_{1}<n_{2}<n_{3}<\ldots$. For any given $n$, we will have $n<n_{\ell}$ for large $\ell$ and we may conclude from (7.8) that

$(7.12) q_{3}^{n}\left[D_{v} h^{l}(v, \mu) \cdot B_{\mu} v+M\left(v, h^{l}(v, \mu)\right)-C_{\mu} h^{l}(v, \mu)-N\left(v, h^{l}(v, \mu)\right)\right]=0$

for large $l$. Notice that we have increased our subscript $i$ from 2 to 3 ; the equation (4.2) holds with $i=2$ also but it is with $i=3$ that we will need it. In fact, hypothesis $i i)$ in section 4 guarantees that the two maps from $H_{2} \times I R$ to $H_{3}$ given by

$$
\begin{aligned}
& (u, \mu) \rightarrow A_{\mu} u \\
& (u, \mu) \rightarrow J(u)
\end{aligned}
$$

are continuous. Assuming in addition that $k \geq 2$, we see then that we may take the limit as $\ell \rightarrow \infty$ in the arguments of (7.12) and conclude that the next equation holds.

$$
q_{3}^{n}\left[D v^{h}(v, \mu) \cdot B_{\mu} v+M(v, h(v, \mu))-C_{\mu} h(v, \mu)-N(v, h(v, \mu))\right]=0
$$

Since $n$ is arbitrary, we see that the $c^{k-1}$ function $h: H^{c} \oplus I R \rightarrow H_{3}^{s}$ a solution in $v_{3}^{k-1}$ of $(6.4-6.5)$. Hence $h$ must be the unique (and so $c^{k}$, solution $f^{\infty}$. 
our conclusion then is that

$f^{1}, f^{2}, f^{3}, \ldots: H^{c} \oplus I R \rightarrow H_{3}^{s}$ must contain a subsequence which converges to $f^{\infty}$ in the $c^{r-1}$ topology. It follows immediately that

$$
\lim _{n \rightarrow \infty} f^{n}=f^{\infty}
$$

in the $c^{r-1}$ topology on $v_{3}^{r}$. That is, the center-unstable manifolds generated by Lanford's argument in our Galerkin approximants approach that of the system (4.8) with $i=3$ or 4 .

\section{The dynamical systems on their center-unstable manifolds}

In any dynamical system in a Hilbert space the flow near a fixed point on its center-unstable manifold, may be identified with a flow near that point, on the tangent space to that manifold at the point. Normally one carries out this identification by means of orthogonal projection onto that tangent space. However, in the case of the systems (6.2) and (7.4), the center-unstable manifolds are the graphs of the globally defined functions $f^{\infty}$ and $f^{n}$ respectively, and it is more convenient to carry out the identification by means of the functions $f^{\infty}$ and $f^{n}$. One sees immediately then that the dynamical system on the center-unstable manifold is equivalent to the syster.

$$
\left(\begin{array}{l}
\dot{v} \\
\dot{\mu}
\end{array}\right)=\left(\begin{array}{c}
B_{\mu} v+M\left(v, f^{\infty}(v, \mu)\right) \\
0
\end{array}\right)=L(v, \mu)
$$

for the system (6.2) and,

$$
\left(\begin{array}{l}
\dot{v} \\
\dot{\mu}
\end{array}\right)=\left(\begin{array}{c}
B_{\mu} v+M\left(v, f^{n}(v, \mu)\right) \\
0
\end{array}\right)=L^{n}(v, \mu)
$$

for the system (7.4). Our hypothesis ii) implies that the function

$$
\text { (8.3) } \quad H^{\mu} \oplus H_{3}^{s}=H_{3}^{\mu} \oplus H_{3}^{s} \stackrel{M}{\rightarrow} H_{4}^{\mu}=H^{\mu}
$$

is $c^{k}$. Consequently, both systems (8.1) and (8.3) are $c^{k}$; moreover, it follows from equation (7.15) that 


$$
\lim _{n \rightarrow \infty} L^{n}=L
$$

in the $c^{k-1}$ topology. Finally, on restricting to the $\varepsilon_{1}$-ball about 0 in $H^{u} \oplus I R$, we obtain a local center-unstable manifold for our original system (2.1); the systern (8.1) now is equivalent to (2.1) on that manifold, and the systems (8.2) approximate it in the $c^{k-1}$ topology.

The proof of Theorem 1 is now complete.

\section{References}

[1] G.D. Birkhoff, "Sur quelques courbes fermees remarquables", Bull. Soc. Math. France 60 (1932), 1-26.

[2] K. Borsuk, The Theory of Shape, (Polish Scientific Publishers, warsaw, 1975).

[3] P. Constantin, C. Foias, and R. Temam, "On the large time Galerkin approximation of the Navier-Stokes equations", SIAM $J$. Nromer. Anal. 21 (1984), 615-634.

[4] J.A. Dutton, "Fundamental theorems of climate theory - some proved, some conjectured", SIAM Rev. 24 (1982), 1-33.

[5] J.A. Dutton and R. Wells, "Topological issues in hydrodynamic predictability", Predictability of Fluid Motions, (AIP Proceedings No. 106, G. Holloway and B.J. West Eds. 1984), 11-43.

[6] J. Guckenheimer, and P. Holmes, Nonlinear Oscillations, Dynamical Systems and Bifurcations of Vector Fields, (Springer Verlag, New York, 1983).

[7] M.W. Hirsch, and B.C. Mazur, Smoothings of Piecewiselinear Manifolds, (Annals of Mathematics Studies 80 Princeton, 1984).

[8] P.E. Kloeden, "Asymptotically stable attracting sets in the Navierstokes equations", BulZ. Austral. Math. Soc. 34 (1986), 37-52.

[9] 0. Ladyshenskaya, The Mathematical Theory of Viscous Incompressible Flow, (Gordon and Breach, New York, 1969).

[10] E.N. Lorenz, "Deterministic non-periodic flow", J. Atmospheric Sci. 20 (1963), 130-141.

[11] J.E. Marsden and M. McCracken, The Hopf Bifurcation and Its Applications, (Springer Verlag, New York, 1976). 
[12] J. Nese, Lyapunov dimension in meteorological finite truncated models, (Masters Thesis, The Pennsylvania State University, 1985).

[13] S. Ostlund, D. Rand, J. Sethna and E. Siggia, "Universal properties of the transition from quasi-periodicity to chaos in dissipative systems", Physica 80 (1983), 303-342.

[14] T. Poston, and I. Stewart, Catastrophe Theory and Its Applications, (Pitman, London 1978).

[15] C.C. Pugh, and M. Shub, "Suspending subshifts", Contributions to Analysis and Geometry, (Johns Hopkins Univ. Press, Baltimore, 1981), 265-275.

[16] R. Temam, Navier-Stokes Equations and Nonlinear Functional Analysis, (CBMS-NSF Regional Conference Series in Applied Mathematics 41 SIAM, 1983).

[17] F. Weissler, "Semilinear evolution equations in Banach spaces", J. Funct. Anal. 32 (1979), 277-296.

[18] R. Williams, "The structure of Lorenz attractors", Publ. Math. Inst. Hantes Etud. Sci. 50 (1979), 73-99.

[19] F.W. Wilson, "Smoothing derivatives of functions and applications", Trans. Amer. Math. Soc. 139 (1969), 413-428.

Department of Mathematics,

College of Science,

The Pennsylvania State University,

215 McAllister Building,

University Park,

Pennsylvania, 16802

United States of America. 